\title{
The association between plasma fibrinogen levels and lung cancer: a meta-analysis
}

\author{
Ke Zhang ${ }^{1 \#}$, Ye Xü ${ }^{1 \#}$, Shanyue Tan ${ }^{1}$, Xueyan Wang ${ }^{1}$, Mulong Du ${ }^{2,3}$, Lingxiang Liu ${ }^{1}$ \\ ${ }^{1}$ Department of Oncology, The First Affiliated Hospital of Nanjing Medical University, Nanjing 210029, China; ${ }^{2}$ Department of Genetic Toxicology, \\ The Key Laboratory of Modern Toxicology of Ministry of Education, School of Public Health, Nanjing Medical University, Nanjing 211166, China; \\ ${ }^{3}$ Department of Biostatistics, Nanjing Medical University, Nanjing 211166, China \\ Contributions: (I) Conception and design: L Liu, M Du; (II) Administrative support: None; (III) Provision of study materials or patients: None; \\ (IV) Collection and assembly of data: K Zhang, Y Xu; (V) Data analysis and interpretation: K Zhang, Y Xu, S Tan, X Wang; (VI) Manuscript writing: \\ All authors; (VII) Final approval of manuscript: All authors. \\ \#These authors contributed equally. \\ Correspondence to: Lingxiang Liu, MD. Department of Oncology, The First Affiliated Hospital of Nanjing Medical University, 300 Guangzhou Road, \\ Nanjing 210029, China. Email: 1lxlau@163.com; Mulong Du, PhD. School of Public Health, Nanjing Medical University, 101 Longmian Avenue, \\ Nanjing 211166, China. Email: drdumulong@njmu.edu.cn.
}

Background: Published studies have presented an inconsistent association between plasma fibrinogen level and poor prognosis or clinicopathological characteristics in lung cancer.

Methods: In the absence of significant quality difference, combined hazard ratios (HRs) and their corresponding 95\% confidence intervals (CIs) were calculated according to overall survival (OS), progression-free survival (PFS) and disease-free survival (DFS). Risk ratio (RR), odds ratio (OR) and standardized mean difference (SMD) with CIs were pooled to appraise the effect of plasma fibrinogen on clinicopathological characteristics. Furthermore, we directly combined the $\mathrm{P}$ values to estimate the association of plasma fibrinogen and tumor size. We adjusted the publication bias using trim-and fill method.

Results: Twenty studies with 6,494 patients were contained in meta-analysis. The pooled data indicated that elevated fibrinogen level associated with poor prognosis in lung cancer. Typically, the pooled HRs were 1.44 (95\% CI, 1.34-1.55), 1.49 (95\% CI, 1.24-1.80) and 1.69 (95\% CI, 1.31-2.17) for OS, PFS and DFS of lung cancer, respectively. In addition, the combined ORs were 1.50 (95\% CI, 1.23-1.84) and 2.01 (95\% CI, 1.66-2.44) for lymph node metastasis and III-IV stage; and the combined RR was 2.15 (95\% CI, 1.11-4.15) for disease control rate (DCR). Moreover, patients with distant metastasis or III-IV stage had significantly higher plasma fibrinogen level (SMD: 0.20, 95\% CI, 0.04-0.36; SMD: 0.31, 95\% CI, 0.18-0.44, respectively).

Conclusions: The summary results indicated that plasma fibrinogen was a marker of prognosis and clinicopathological characteristics in lung cancer.

Keywords: Plasma fibrinogen; lung cancer; prognosis; clinicopathological characteristics; meta-analysis

Submitted Oct 14, 2019. Accepted for publication Nov 06, 2019.

doi: $10.21037 /$ jtd.2019.11.13

View this article at: http://dx.doi.org/10.21037/jtd.2019.11.13

\section{Introduction}

Lung cancer was considered to be the leading cause of cancerrelated mortality (1). There were 1.8 million new lung cancer cases and 1.6 million cancer-related deaths worldwide (2). In spite of great advances in diagnosis and therapy, 5-year survival rates are only $18 \%$ for lung cancer (3). Independent prognostic factors, including disease stage, performance status, age, sex, weight loss and KRAS mutation, have been identified (4-8). However, there is a lack of blood biochemical indicators for the prognosis and clinicopathological 
characteristics in patients suffering from lung cancer.

Fibrinogen, a easily measured biochemical indicator, is a kind of the principal acute phase proteins generated by the liver (9). The activation of coagulation and fibrinolysis is encountered among malignancy and about $90 \%$ of cancer patients with metastatic disease and $50 \%$ of cancer patients have abnormal coagulation parameters (10). Moreover, high plasma fibrinogen level is frequently observed in cancer patients and influence metastatic potential $(11,12)$. Several meta-analysis indicated that elevated plasma fibrinogen associated with poor prognosis (overall survival, OS; progression-free survival, PFS; disease-free survival, DFS) in epithelial ovarian, digestive cancer $(13,14)$. Moreover, elevated plasma fibrinogen level in lung cancer implied short survival (15). Then, anticoagulant therapy would be valuable for patients with lung cancer. However, conflicting data were reported $(16,17)$.

Therefore, we conducted a meta-analysis to assess the prognostic significance of fibrinogen in patients with lung cancer.

\section{Methods}

\section{Search strategy}

A systemic search in PubMed, Web of science, EMBASE and the Cochrane Central Register of Controlled Trials databases were employed to identify all articles published up to September 2018, using the items "fibrinogen", "hyperfibrinogen", "fibrinogenemia", "predictive", "recurrence", "relapse", "prognosis", "survival", "lung cancer" and "lung neoplasms". In addition, we manually searched abstracts of the World Lung Cancer Conference, the American Society of Clinical Oncology (ASCO) and the European Society of Medical Oncology (ESMO) to identify unpublished studies.

\section{Selection criteria}

Eligible studies included in the meta-analysis had to meet the following criteria: (I) assessment of the association of plasma fibrinogen and lung cancer prognosis or clinicopathological characteristics; (II) the value of plasma fibrinogen detection before chemotherapy; (III) only including the most recent and informative studies if duplicate articles were from the same participants; (IV) full text articles in English or Chinese language were retained.

\section{Data extraction}

Data from the included studies were extracted independently by two investigators ( $\mathrm{K}$ Zhang and $\mathrm{Y} \mathrm{Xu}$ ). The information, including first author, year of publication, patient source, number of patients, histology, disease stage, cut-off value and outcomes, was extracted. Two reviewers (K Zhang and $\mathrm{Y} \mathrm{Xu}$ ) determined study eligibility independently and any disagreements were solved after discussion.

\section{Quality assessment}

The STrengthening the Reporting of OBservational studies in Epidemiology (STROBE) checklist was used to assess the quality of these studies for cohort studies (18). The overall score assessed the following dimensions of methodology, grouped into eight main categories: (I) hypothesis and/or objective(s) stated; (II) tumor stage clearly described; (III) clear description of eligibility criteria; (IV) patients with inflammatory disease or coagulation disorders excluded; (V) predictors and outcome(s) clearly predefined; (VI) confounders considered in multivariate analysis; (VII) long enough for outcomes to occur; (VIII) bias and limitations considered. Each category had 1 point, and then an overall maximum theoretical score was 8 points. Finally, the scores were expressed as numbers from 0 to 8 , and better methodological quality was provided with higher values (Table S1).

\section{Definition of outcomes}

The primary outcomes were the OS, PFS, DFS or clinicopathological characteristics, including disease control rate (DCR), objective response rate (ORR), tumor stage, lymph node metastasis, distant metastasis, stage, differentiation, Eastern Cooperative Oncology Group (ECOG), histology and tumor size. Then, we conducted stratified analysis by histology type, stage, region of study and cut-off value for plasma fibrinogen. The effective value of OS, PFS and DFS was determined by the combination of HR and $95 \%$ confidence interval (CI). Estimated value was conducted indirectly from Kaplan-Meier plot using the methods reported by Tierney et al. (19) if a direct report of HR and corresponding 95\% CI was not available. To assess the reliability, we calculated pooled HRs in studies with multivariate analysis. We employed relative risk (RR), odds ratio (OR) and standardized mean difference (SMD) to evaluate clinicopathological characteristics, including 


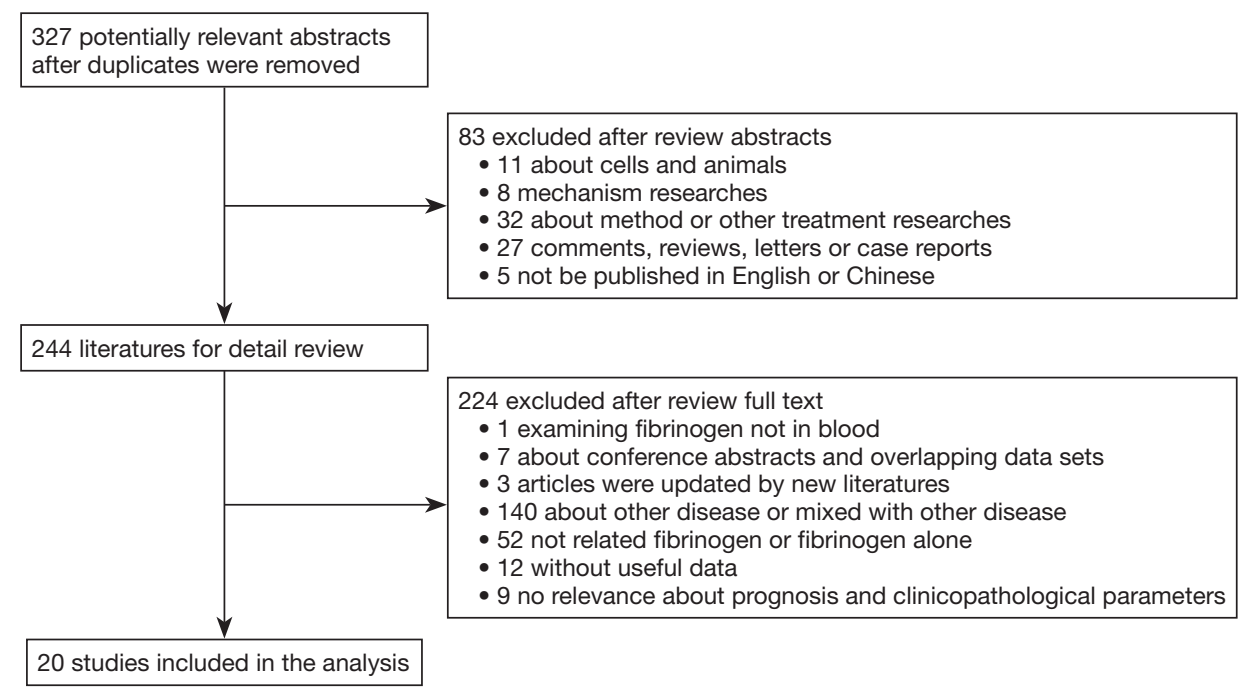

Figure 1 Flow chart of the search strategy and study selection.

DCR, ORR, tumor stage, lymph node metastasis, distant metastasis, stage, differentiation, ECOG and histology.

\section{Statistical analysis}

Significant heterogeneity was identified if $\mathrm{I}^{2}$ more than $50 \%$ and $\mathrm{P}<0.05$ with the Cochran's Q-test and $\mathrm{I}^{2}$, then the random-effects model was used. Otherwise, it was calculated by the fixed-effects model without significant heterogeneity. The combination of the estimated risk was calculated by the $\mathrm{Z}$ test, and $\mathrm{P}<0.05$ was considered statistically. Publication bias, assessed by rank correlation and linear regression method, was adjusted by the trim and fill method if publication bias existed $(\mathrm{P}<0.05)$. The $\mathrm{R}$ Statistical Software (version 3.5.1) was performed for statistical analyses. The "meta" package was applied to generate the pooled estimates and publication bias assessment. The "forestplot" package was used to produce forest plot. All $\mathrm{P}$ values were two sided.

\section{Results}

\section{Trial flow}

The result of the study searching process depicts in Figure 1. A total of 327 potentially relevant articles were identified, and 83 of them were excluded for the following reasons: 11 cell lines or animals, 8 mechanism researches, 32 method or other treatment researches, 27 comments, reviews, letters or case reports and 5 published in non-English and nonChinese language. After reviewing full-text carefully, 20 articles were included in the analysis.

\section{Study characteristics}

From 1997 to 2018, a total of 6,494 patients suffering from lung cancer were enrolled into the meta-analysis, the median sample size was 235 (ranged from 58 to 856). In this analysis, cut-off value of plasma fibrinogen was $4.0 \mathrm{~g} / \mathrm{L}$ in eight studies. Besides, 16 (15-17,20-32), four $(22,26,29,31)$ and 3 studies $(25,29,33)$ were estimated by HR and corresponding $95 \%$ CI for OS, PFS and DFS, respectively. Two studies were evaluated by OR for histology $(29,33)$, differentiation $(29,33)$, chemotherapy response (DCR and ORR) $(26,31)$ and performance status $(26,33)$ and four $(24,29,33,34)$ and three articles $(24,29,33)$ for stage and lymph node metastasis, respectively. Furthermore, SMD was applied to appraise the effect of fibrinogen on clinicopathological characteristics, in which four studies $(16,31,35,36)$ for histology and three for distant metastasis $(16,24,35)$, tumor stage $(24,35,36)$ and two for stage $(24,35)$ (Table 1). Fourteen studies (15,20-32) investigated elevated plasma fibrinogen as an indicator of poor OS, and the other two studies $(16,17)$ showed no significant impact on OS. Moreover, three studies $(22,26,29)$ indicated that elevated plasma fibrinogen as a marker for poor PFS, and only one study (31) showed no effect on PFS. 
Table 1 Characteristics of studies included from the meta-analysis

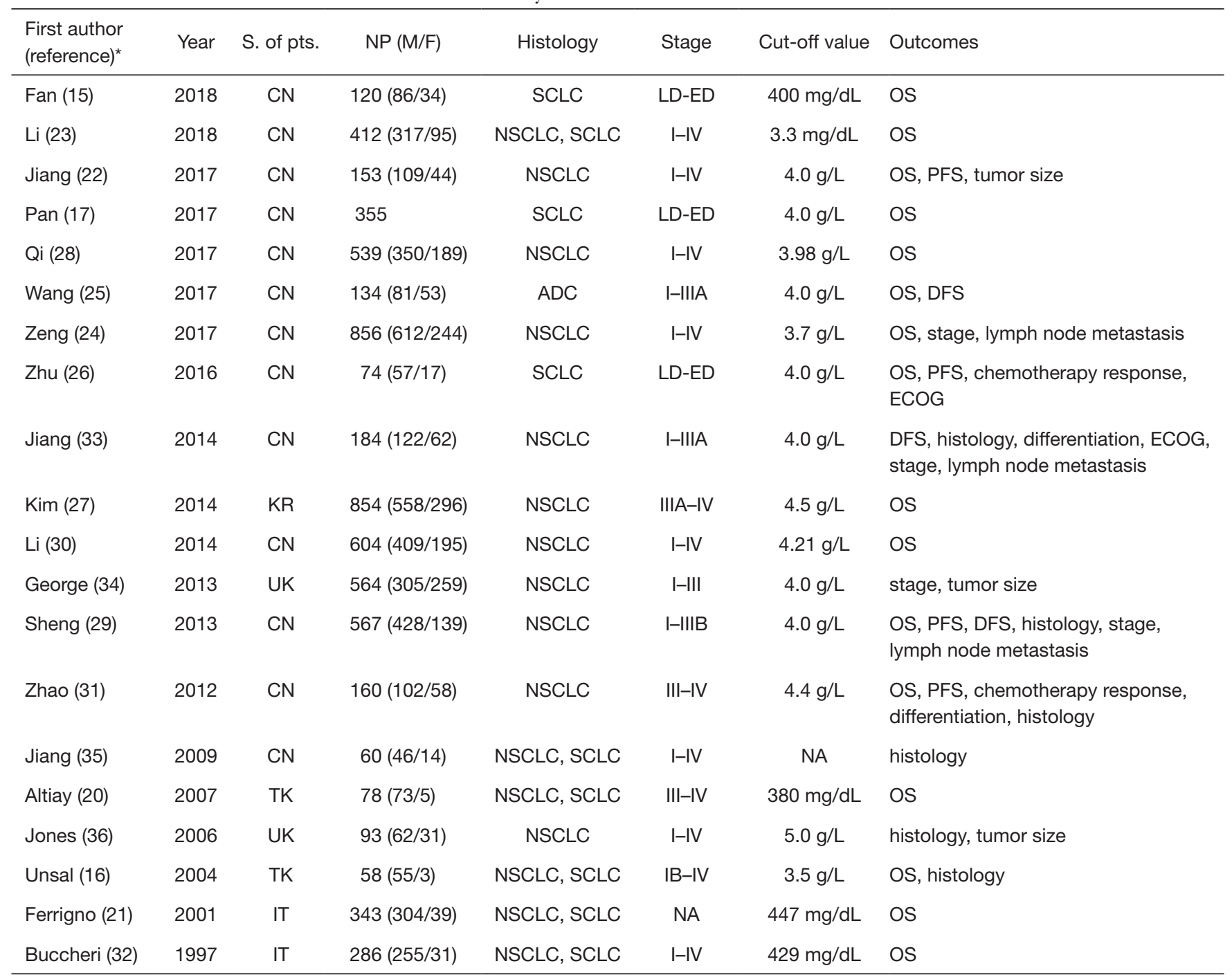

*, references as described in manuscript; S. of pts., source of patients; NP, number of patients; TK, Turkey; CN, China; IT, Italy; KR, Korea; UK, United Kingdom; NSCLC, non-small cell lung cancer; SCLC, small cell lung cancer; OS, overall survival; PFS, progression-free survival; DFS, disease-free survival; ECOG, Eastern Cooperative Oncology Group; NA, not available.

\section{Meta-analysis of OS, PFS and DFS}

Figures 2 and $S 1-S 3$ shows results of each meta-analysis. The overall studies were systematic analyzed, with the combined HR 1.44 (95\% CI $=1.34-1.55), 1.49$ (95\% CI $=1.24-1.80)$ and $1.69(95 \% \mathrm{CI}=1.31-2.17)$ for OS (Figure $2 A)$, PFS (Figure $2 B$ ) and DFS (Figure 2C), respectively. In addition, the association was also validated in studies with multivariable analyses (HR=1.48, 95\% CI $=1.36-1.61$ for OS, Figure S1A; $\mathrm{HR}=1.53,95 \% \mathrm{CI}=1.11-2.12$ for PFS, Figure S1B). The results were consistent with analysis in overall studies. The pooled HR was 1.46 (95\% CI $=1.34-1.60)$ in studies excluded inflammatory disease (Figure S2). As shown in Figure 3, the results implied that elevated plasma fibrinogen level effected on poor OS in subgroup analyses about histology type, stage, region of study and cut-off value for plasma fibrinogen. The combined HR was 1.54 (95\% CI $=1.40-1.69)$ for NSCLC, $1.25(95 \% \mathrm{CI}=1.05-1.48)$ for SCLC, $1.99(95 \% \mathrm{CI}=1.50$ 2.65) for stage I-III, $1.52(95 \% \mathrm{CI}=1.36-1.71)$ for stage III$\mathrm{IV}, 1.45$ (95\% CI $=1.34-1.57), 1.42(95 \% \mathrm{CI}=1.21-1.67), 1.48$ (95\% CI $=1.35-1.63), 1.39$ (95\% CI $=1.25-1.56)$ for Asian, non-Asian, China, non-China, respectively. The pooled HRs were $1.40(95 \% \mathrm{CI}=1.22-1.59), 1.47(95 \% \mathrm{CI}=1.35-1.59)$ 


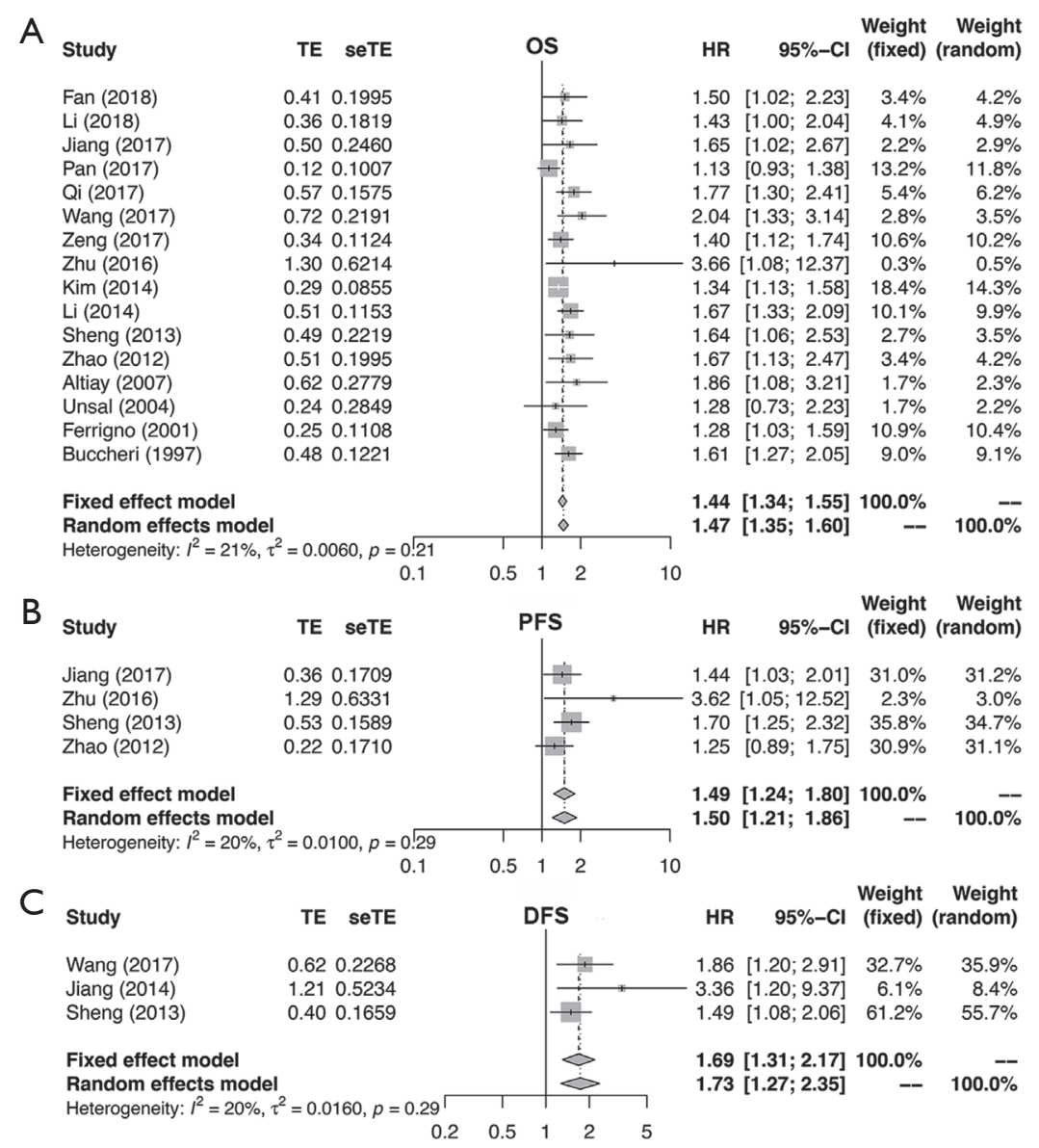

Figure 2 Meta-analysis for assessing the association between plasma fibrinogen and OS (A), PFS (B), DFS (C) of lung cancer. OS, overall survival; PFS, progression-free survival; DFS, disease-free survival.

\section{Overall survival}

\begin{tabular}{|c|c|c|c|c|c|c|c|}
\hline Subgroups & NS & NP & & HR (95\% Cl) & $\operatorname{tau} \wedge 2$ & I-squared & $\mathbf{P}$ \\
\hline All patients & 16 & 5593 & - & $1.44(1.34-1.55)$ & 0.01 & 0.21 & 0.21 \\
\hline \multicolumn{8}{|l|}{ Histology } \\
\hline NSCLC & 9 & 4203 & $\vdash \rightarrow$ & $1.54(1.40-1.69)$ & 0.00 & 0.00 & 0.47 \\
\hline SCLC & 4 & 607 & $\longmapsto$ & $1.25(1.05-1.48)$ & 0.05 & 0.46 & 0.14 \\
\hline \multicolumn{8}{|l|}{ Stage } \\
\hline I-III & 3 & 948 & $\longmapsto$ & $1.99(1.50-2.65)$ & 0.02 & 0.24 & 0.27 \\
\hline III-IV & 6 & 2060 & $\vdash \longrightarrow$ & $1.52(1.36-1.71)$ & 0.01 & 0.31 & 0.20 \\
\hline \multicolumn{8}{|l|}{ Region } \\
\hline Asian & 14 & 4964 & $\vdash-1$ & $1.45(1.34-1.57)$ & 0.01 & 0.24 & 0.20 \\
\hline Non-Asian & 2 & 629 & $\longmapsto \longrightarrow$ & $1.42(1.21-1.67)$ & 0.01 & 0.48 & 0.16 \\
\hline China & 11 & 3974 & ・ー & $1.48(1.35-1.63)$ & 0.01 & 0.33 & 0.13 \\
\hline Non-China & 5 & 1619 & $\vdash \multimap$ & $1.39(1.25-1.56)$ & 0.00 & 0.00 & 0.50 \\
\hline \multicolumn{8}{|c|}{ Cut-off value } \\
\hline $4.0 \mathrm{~g} / \mathrm{L}$ & 7 & 1678 & $\longmapsto \longrightarrow$ & $1.40(1.22-1.59)$ & 0.03 & 0.46 & 0.08 \\
\hline non-4.0g/L & 10 & 4190 & 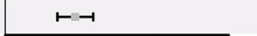 & $1.47(1.35-1.59)$ & 0.00 & 0.00 & 0.58 \\
\hline
\end{tabular}

Figure 3 Meta-analysis in subgroups about histology type, stage, region of study and cutoff value. 


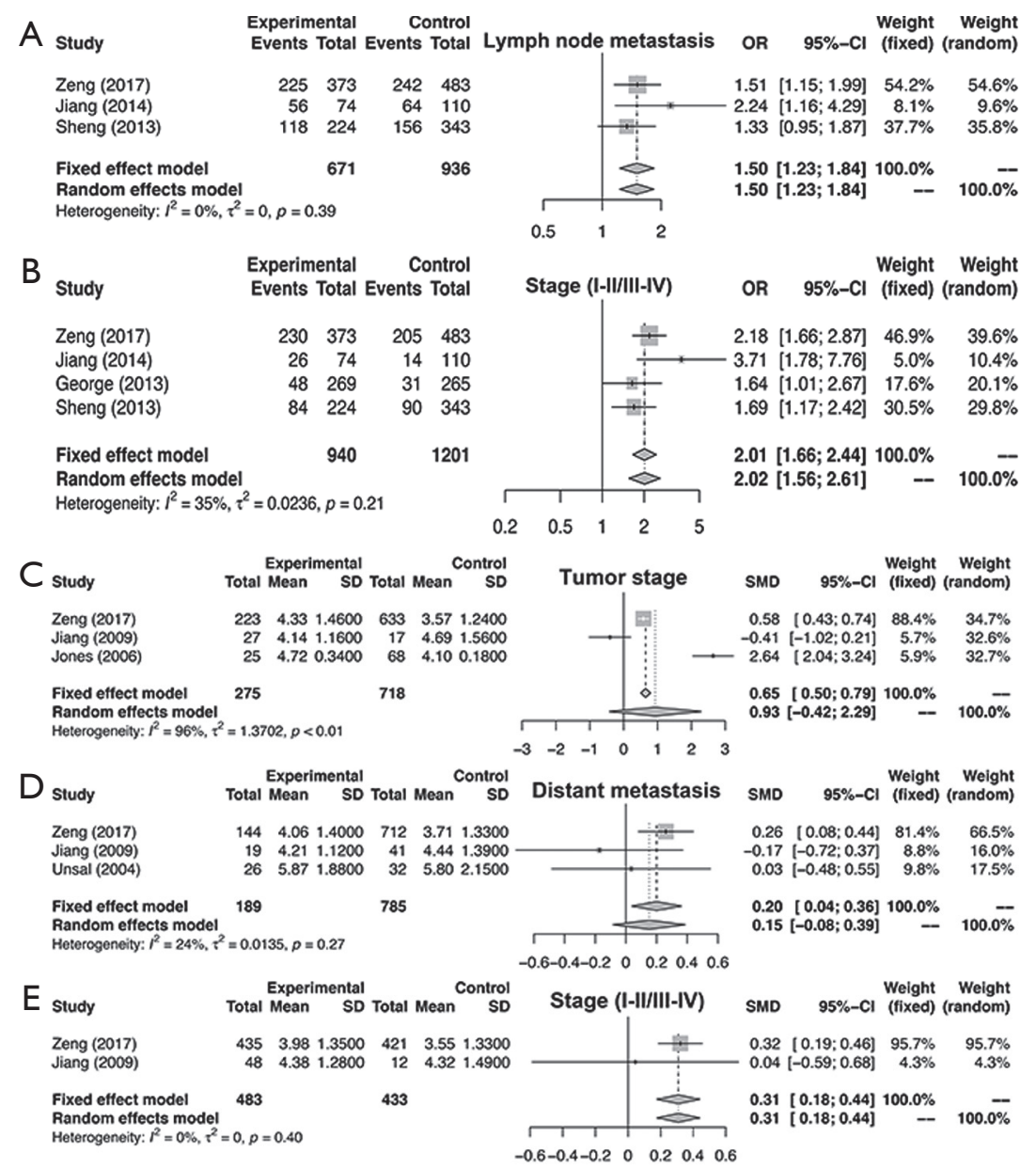

Figure 4 Meta-analysis about TNM stage in lymph node metastasis (A) and stage (B) estimated by odds ratio; tumor stage (C), distant metastasis $v s$. no distant metastasis (D), stage III-IV vs. stage I-II (E) estimated by standardized mean difference.

for studies with cut-off value equal to $4 \mathrm{~g} / \mathrm{L}$ and was not $4 \mathrm{~g} / \mathrm{L}$.

\section{Meta-analysis of clinicopathological characteristics}

Similarly, the meta-analysis indicated that fibrinogen associated with clinicopathological characteristics. The pooled RRs and corresponding 95\% CI were 2.15 (95\% CI, 1.11-4.15) and 1.34 (95\% CI, 0.68-2.62) for DCR (Figure $S 3 A$ ) and ORR (Figure $S 3 B$ ), respectively. In meta-analysis about TNM stage, the combined ORs were 1.50 (95\% CI, 1.23-1.84) and 2.01 (95\% CI, 1.66-2.44) lymph node metastasis (Figure $4 A$ ) and stage III-IV vs. stage I-II (Figure 4B), respectively. Moreover, the SMDs were 0.93 (95\% CI, -0.42 to 2.29$), 0.20$ (95\% CI, 0.04-0.36) and 0.31 (95\% CI, 0.18-0.44) for tumor stage (Figure 4C), distant metastasis vs. no distant metastasis (Figure 4D) and stage III-IV vs. stage I-II (Figure $4 E$ ). No significance was found in poor differentiation (Figure $S 4 A$ ), and performance status (Figure $S 4 B$ ). In addition, the results showed no differences in histology (NSCLC and SCLC, adenocarcinoma and squamous cell carcinoma) (Figure $S 5 A, B, C$ ). The $\mathrm{P}$ value directly combined implied that plasma fibrinogen might affect tumor size $\left(\mathrm{P}=2.82 \times 10^{-5}\right)$.

\section{Test of heterogeneity}

We analyzed the heterogeneity in all included studies between fibrinogen and the prognosis of lung cancer. The heterogeneity, sixteen studies for OS, four studies for PFS, three studies for DFS, were evaluated in a fixed-effects model. Moreover, the heterogeneity of included studies 
was $21 \%, 20 \%$ and $20 \%$ with the $\mathrm{I}^{2}$ value, respectively. Heterogeneity was no significance in the OS subgroup analysis (Figure $2 A, B, C$ ).

\section{Publication bias}

As shown in Figures S6,S7, the rank correlation and linear regression method were used to evaluate publication bias of the meta-analysis. We found a significant funnel plot asymmetry, with $\mathrm{P}=0.011$ in the linear regression test and $\mathrm{P}=0.072$ in the rank correlation test, demonstrating the existence of publication bias in OS. However, in PFS and DFS, no significant funnel plot asymmetry was observed, with $\mathrm{P}=0.323$ and $\mathrm{P}=0.125$ in the linear regression test, $\mathrm{P}=1.000$ and $\mathrm{P}=0.117$ in the rank correlation test. Furthermore, we used a trim-and-fill method to adjust the bias, which was developed by Duval and Tweedie (37). The adjusted result was similar with our results ( $\mathrm{HR}=1.39$, 95\% CI, 1.30-1.49) in OS (Figure S7).

\section{Discussion}

Elevated pretreatment plasma fibrinogen predicted poor prognosis by meta-analysis in epithelial ovarian, digestive cancer $(13,14)$. However, it was inconsistent whether fibrinogen was a prognosis factor in lung cancer. To our knowledge, this is the first systemic analysis about the association between fibrinogen and lung cancer prognosis. We performed a comprehensive analysis including 6,494 patients. It was showed that elevated pretreatment plasma fibrinogen levels could predict poor survival of lung cancer. Subgroup analysis also validated the result, according to region of study, histology type, cut-off value for plasma fibrinogen and HR estimated method for survival. Therefore, fibrinogen could play as a predictive factor of OS, PFS and DFS.

In addition, we also analyzed the association between fibrinogen and clinicopathological characteristics. We observed that elevated plasma fibrinogen affected TNM stage excluding tumor stage due to significant heterogeneity in lung cancer patients. However, plasma fibrinogen did not associate with differentiation and performance status and histology neither ADC vs. SCC nor NSCLC vs. SCLC. Due to limited sample size, the results of DCR and ORR were inconsistent. Considering the smoking was as a risk factor in lung cancer (38), we further found that smoking may affect plasma fibrinogen level and the pooled OR was
2.06 (95\% CI, 1.64-2.59) analyzed in two studies $(24,29)$.

In terms of fibrinogen in the prognosis of lung cancer, the evidence supported our conclusion as follow. Stromaderived Fibrinogen-like Protein 2 promote tumor growth by activating cancer-associated fibroblasts in the tumor microenvironment in lung cancer (39). Meanwhile, the study revealed that fibrinogen levels are significantly high among different pathologic types of lung cancer patients (40). In addition, elevated fibrinogen was associated with performance status after lung cancer resection surgery (41). Moreover, high plasma fibrinogen was associated with poorer lymph node status, recurrence and advanced pathologic stage (33). Due to the close relationships between fibrinogen and worse clinical pathological features of lung cancer, the relation between fibrinogen and poor survival of lung cancer was understandable. Plasma fibrinogen level of lung cancer patients reduced obviously after surgery indicated the higher tumor burden the elevated plasma fibrinogen (33). Therefore, the plasma fibrinogen in pretreatment lung cancer may affect prognosis and metastasis by tumor microenvironment.

Though our study provided a relatively convincing conclusion that fibrinogen could play as a prognosis biomarker of lung cancer, certain inevitable limitations should be stated: (I) we did not exclude the study that reported the relation between serum fibrinogen and lung cancer prognosis. Fibrinogen do not exist in serum and we recognized that author was clerical error; (II) studies was excluded due to unavailable data for meta-analysis which may lead to publication bias $(35,42,43)$. Moreover, this metaanalysis indicated existence of publication bias, however, we searched conference abstracts and the heterogeneity test and the trim and fill method demonstrated the conclusion was stable; (III) the therapeutic regimen was different may lead to bias. So, conducting high-quality research is necessary.

Overall, our meta-analysis indicated that pretreatment may as a poor prognosis factor in lung cancer. Moreover, plasma fibrinogen associated with TNM stage rather than ORR. Nonetheless, a well-designed prospective study with a large sample to confirm our results and cover the above limitations is essential.

\section{Acknowledgments}

Funding: This study was supported by National Natural Science Foundation of China (81472782); the National Key R\&D Program of China (2017YFC0908200). 


\section{Footnote}

Conflicts of Interest: The authors have no conflicts of interest to declare.

Ethical Statement: The authors are accountable for all aspects of the work in ensuring that questions related to the accuracy or integrity of any part of the work are appropriately investigated and resolved.

\section{References}

1. Torre LA, Siegel RL, Ward EM, et al. Global Cancer Incidence and Mortality Rates and Trends--An Update. Cancer Epidemiol Biomarkers Prev 2016;25:16-27.

2. Ferlay J, Soerjomataram I, Dikshit R, et al. Cancer incidence and mortality worldwide: sources, methods and major patterns in GLOBOCAN 2012. Int J Cancer 2015;136:E359-86.

3. Mantovani G, Madeddu C, Gramignano G, et al. Subcutaneous interleukin-2 in combination with medroxyprogesterone acetate and antioxidants in advanced cancer responders to previous chemotherapy: phase II study evaluating clinical, quality of life, and laboratory parameters. J Exp Ther Oncol 2003;3:205-19.

4. Paesmans M, Sculier JP, Libert P, et al. Prognostic factors for survival in advanced non-small-cell lung cancer: univariate and multivariate analyses including recursive partitioning and amalgamation algorithms in 1,052 patients. The European Lung Cancer Working Party. J Clin Oncol 1995;13:1221-30.

5. Albain KS, Crowley JJ, LeBlanc M, et al. Determinants of improved outcome in small-cell lung cancer: an analysis of the 2,580-patient Southwest Oncology Group data base. J Clin Oncol 1990;8:1563-74.

6. Foster NR, Mandrekar SJ, Schild SE, et al. Prognostic factors differ by tumor stage for small cell lung cancer: a pooled analysis of North Central Cancer Treatment Group trials. Cancer 2009;115:2721-31.

7. Finkelstein DM, Ettinger DS, Ruckdeschel JC. Longterm survivors in metastatic non-small-cell lung cancer: an Eastern Cooperative Oncology Group Study. J Clin Oncol 1986;4:702-9.

8. Eberhard DA, Johnson BE, Amler LC, et al. Mutations in the epidermal growth factor receptor and in KRAS are predictive and prognostic indicators in patients with non-small-cell lung cancer treated with chemotherapy alone and in combination with erlotinib. J Clin Oncol
2005;23:5900-9.

9. Tennent GA, Brennan SO, Stangou AJ, et al. Human plasma fibrinogen is synthesized in the liver. Blood 2007;109:1971-4.

10. Repetto O, De Re V. Coagulation and fibrinolysis in gastric cancer. Ann N Y Acad Sci 2017;1404:27-48.

11. Allin KH, Bojesen SE, Nordestgaard BG. Inflammatory biomarkers and risk of cancer in 84,000 individuals from the general population. Int J Cancer 2016;139:1493-500.

12. Staton CA, Brown NJ, Lewis CE. The role of fibrinogen and related fragments in tumour angiogenesis and metastasis. Expert Opin Biol Ther 2003;3:1105-20.

13. Luo Y, Kim HS, Kim M, et al. Elevated plasma fibrinogen levels and prognosis of epithelial ovarian cancer: a cohort study and meta-analysis. J Gynecol Oncol 2017;28:e36.

14. Lin Y, Liu Z, Qiu Y, et al. Clinical significance of plasma D-dimer and fibrinogen in digestive cancer: A systematic review and meta-analysis. Eur J Surg Oncol 2018;44:1494-503.

15. Fan S, Guan Y, Zhao G, et al. Association between plasma fibrinogen and survival in patients with small-cell lung carcinoma. Thorac Cancer 2018;9:146-51.

16. Unsal E, Atalay F, Atikcan S, et al. Prognostic significance of hemostatic parameters in patients with lung cancer. Respir Med 2004;98:93-8.

17. Pan H, Shi X, Xiao D, et al. Nomogram prediction for the survival of the patients with small cell lung cancer. J Thorac Dis 2017;9:507-18.

18. von Elm E, Altman DG, Egger M, et al. The Strengthening the Reporting of Observational Studies in Epidemiology (STROBE) Statement: guidelines for reporting observational studies. Int J Surg 2014;12:1495-9.

19. Tierney JF, Stewart LA, Ghersi D, et al. Practical methods for incorporating summary time-to-event data into metaanalysis. Trials 2007;8:16.

20. Altiay G, Ciftci A, Demir M, et al. High Plasma d-dimer Level is Associated with Decreased Survival in Patients with Lung Cancer. Clin Oncol (R Coll Radiol) 2007;19:494-8.

21. Ferrigno D, Buccheri G, Ricca I. Prognostic significance of blood coagulation tests in lung cancer. Eur Respir J 2001;17:667-73.

22. Jiang LY, Zhang XF, Ding FB, et al. Prognostic implications of plasma fibrinogen and serum C-reactive protein levels in non-small cell lung cancer resection and survival. Trop J Pharm Res 2017;16:665-72.

23. Li SQ, Jiang YH, Lin J, et al. Albumin-to-fibrinogen 
ratio as a promising biomarker to predict clinical outcome of non-small cell lung cancer individuals. Cancer Med 2018;7:1221-31.

24. Zeng Q, Xue N, Dai D, et al. A Nomogram based on Inflammatory Factors C-Reactive Protein and Fibrinogen to Predict the Prognostic Value in Patients with Resected Non-Small Cell Lung Cancer. J Cancer 2017;8:744-53.

25. Wang YQ, Zhi QJ, Wang XY, et al. Prognostic value of combined platelet, fibrinogen, neutrophil to lymphocyte ratio and platelet to lymphocyte ratio in patients with lung adenosquamous cancer. Oncol Lett 2017;14:4331-8.

26. Zhu LR, Li J, Chen P, et al. Clinical significance of plasma fibrinogen and D-dimer in predicting the chemotherapy efficacy and prognosis for small cell lung cancer patients. Clin Transl Oncol 2016;18:178-88.

27. Kim KH. Prognostic significance of initial platelet counts and fibrinogen level in advanced non-small cell lung cancer. J Korean Med Sci 2014;29:507-11.

28. Qi Y, Fu J. Research on the coagulation function changes in non small cell lung cancer patients and analysis of their correlation with metastasis and survival. J BUON 2017;22:462-7.

29. Sheng L, Luo M, Sun X, et al. Serum fibrinogen is an independent prognostic factor in operable nonsmall cell lung cancer. Int J Cancer 2013;133:2720-5.

30. Li Y, Wei S, Wang J, et al. Analysis of the factors associated with abnormal coagulation and prognosis in patients with non-small cell lung cancer. Zhongguo Fei Ai Za Zhi 2014;17:789-96.

31. Zhao J, Zhao M, Jin B, et al. Tumor response and survival in patients with advanced non-small-cell lung cancer: the predictive value of chemotherapy-induced changes in fibrinogen. BMC Cancer 2012;12:330.

32. Buccheri G, Ferrigno D, Ginardi C, et al. Haemostatic abnormalities in lung cancer: prognostic implications. Eur J Cancer 1997;33:50-5.

33. Jiang HG, Li J, Shi SB, et al. Value of fibrinogen and D-dimer in predicting recurrence and metastasis after

Cite this article as: Zhang $\mathrm{K}, \mathrm{Xu} \mathrm{Y}$, Tan S, Wang X, Du M, Liu L. The association between plasma fibrinogen levels and lung cancer: a meta-analysis. J Thorac Dis 2019;11(11):4492-4500. doi: $10.21037 /$ jtd.2019.11.13 radical surgery for non-small cell lung cancer. Med Oncol 2014;31:22.

34. George RS, Smith MO, Sharkey AJ, et al. Is plasma fibrinogen a novel independent prognostic factor in patients undergoing surgery for non-small cell lung cancer? Lung Cancer 2013;79:S27.

35. Jiang Z, Sang H, Ge H, et al. The study of prethrombotic state in patients with lung cancer. Zhongguo Fei Ai Za Zhi 2009;12:44-8.

36. Jones JM, McGonigle NC, McAnespie M, et al. Plasma fibrinogen and serum $\mathrm{C}$-reactive protein are associated with non-small cell lung cancer. Lung Cancer 2006;53:97-101.

37. Duval S, Tweedie R. Trim and fill: A simple funnel-plotbased method of testing and adjusting for publication bias in meta-analysis. Biometrics 2000;56:455-63.

38. Hong S, Mok Y, Jeon C, et al. Tuberculosis, smoking and risk for lung cancer incidence and mortality. Int J Cancer 2016;139:2447-55.

39. Zhu Y, Zhang L, Zha H, et al. Stroma-derived Fibrinogenlike Protein 2 Activates Cancer-associated Fibroblasts to Promote Tumor Growth in Lung Cancer. Int J Biol Sci 2017;13:804-14.

40. Nakano K, Sugiyama K, Satoh H, et al. Risk factors for disseminated intravascular coagulation in patients with lung cancer. Thorac Cancer 2018;9:931-8.

41. Araujo AS, Nogueira IC, Gomes Neto A, et al. The impact of lung cancer resection surgery on fibrinogen and C-reactive protein and their relationship with patients outcomes: A prospective follow up study. Cancer Biomark 2016;16:47-53.

42. Tas F, Kilic L, Serilmez M, et al. Clinical and prognostic significance of coagulation assays in lung cancer. Respir Med 2013;107:451-7.

43. George R, Smith M, Sharkey A, et al. Is plasma fibrinogen a novel independent prognostic factor in patients undergoing surgery for non-small cell lung cancer? Interact Cardiovasc Thorac Surg 2013;79:S27. 


\section{Supplementary}

Table S1 The score of included studies for meta-analysis

\begin{tabular}{|c|c|c|c|c|c|c|c|c|c|c|}
\hline Study (reference) ${ }^{*}$ & Year & A & B & C & D & E & $\mathrm{F}$ & G & $\mathrm{H}$ & Quality score $(0-8)$ \\
\hline Fan (15) & 2018 & Y & Y & Y & Y & Y & Y & $\mathrm{N}$ & $\mathrm{Y}$ & 7 \\
\hline Li (23) & 2018 & Y & Y & $Y$ & $Y$ & Y & Y & Y & $\mathrm{N}$ & 7 \\
\hline Jiang (22) & 2017 & Y & Y & Y & Y & Y & $\mathrm{N}$ & Y & $\mathrm{N}$ & 6 \\
\hline Pan (17) & 2017 & Y & Y & $Y$ & N & Y & Y & $\mathrm{N}$ & $\mathrm{N}$ & 5 \\
\hline Qi (28) & 2017 & Y & $\mathrm{N}$ & $Y$ & $Y$ & $\mathrm{~N}$ & Y & Y & $\mathrm{N}$ & 5 \\
\hline Wang (25) & 2017 & Y & $\mathrm{N}$ & $\mathrm{Y}$ & $\mathrm{Y}$ & $\mathrm{N}$ & $\mathrm{N}$ & Y & $\mathrm{N}$ & 4 \\
\hline Zeng (24) & 2017 & Y & Y & $Y$ & $Y$ & Y & Y & $\mathrm{N}$ & $\mathrm{N}$ & 6 \\
\hline Zhu (26) & 2016 & Y & Y & $Y$ & Y & Y & Y & $\mathrm{N}$ & $\mathrm{N}$ & 6 \\
\hline Jiang (33) & 2014 & Y & N & $Y$ & $Y$ & Y & Y & N & $\mathrm{N}$ & 5 \\
\hline Kim (27) & 2014 & Y & Y & Y & Y & Y & Y & Y & $\mathrm{N}$ & 7 \\
\hline Li (30) & 2014 & Y & Y & $Y$ & N & Y & Y & Y & $\mathrm{Y}$ & 7 \\
\hline George (34) & 2013 & Y & Y & N & N & Y & N & $\mathrm{N}$ & $\mathrm{N}$ & 3 \\
\hline Sheng (29) & 2013 & Y & Y & Y & Y & Y & Y & Y & $\mathrm{N}$ & 7 \\
\hline Zhao (31) & 2012 & Y & Y & $Y$ & $Y$ & Y & Y & Y & $\mathrm{N}$ & 7 \\
\hline Jiang (35) & 2009 & Y & Y & $Y$ & $Y$ & Y & N & $\mathrm{N}$ & $\mathrm{N}$ & 5 \\
\hline Altiay (20) & 2007 & Y & $\mathrm{N}$ & $Y$ & $Y$ & Y & Y & Y & $\mathrm{N}$ & 6 \\
\hline Jones (36) & 2006 & Y & Y & $\mathrm{N}$ & N & Y & Y & $\mathrm{N}$ & $\mathrm{N}$ & 4 \\
\hline Unsal (16) & 2004 & Y & Y & $Y$ & $Y$ & Y & N & Y & $\mathrm{N}$ & 6 \\
\hline Ferrigno (21) & 2001 & Y & N & $\mathrm{N}$ & Y & Y & N & Y & $\mathrm{N}$ & 4 \\
\hline Buccheri (32) & 1997 & Y & $\mathrm{N}$ & $\mathrm{Y}$ & $\mathrm{N}$ & $\mathrm{Y}$ & $\mathrm{Y}$ & $\mathrm{N}$ & $\mathrm{N}$ & 4 \\
\hline
\end{tabular}

*, references as described in manuscript; A, hypothesis and/or objective(s) stated; B, tumor stage clearly described; C, clear description of eligibility criteria; D, patients with inflammatory disease or coagulation disorders excluded; E, predictors and outcome(s) clearly predefined; F, confounders considered in multivariate analysis; $\mathrm{G}$, long enough for outcomes to occur; $\mathrm{H}$, bias and limitations considered; $\mathrm{Y}$, yes; N, no. 


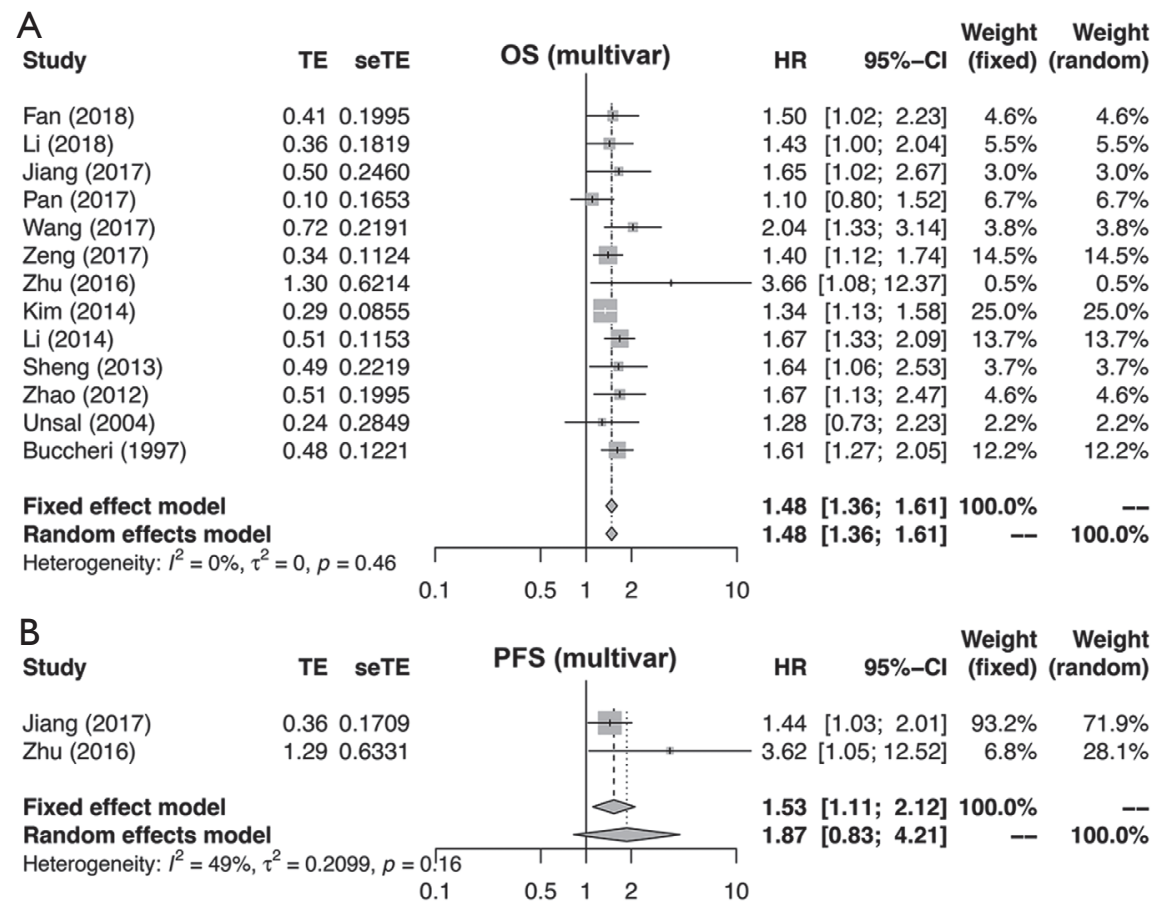

Figure S1 Meta-analysis for assessing the association between plasma fibrinogen and OS (A) and PFS (B) in studies with multivariable analyses in lung cancer. OS, overall survival; PFS, progression-free survival; multivar, analysis in studies with multivariable analyses.

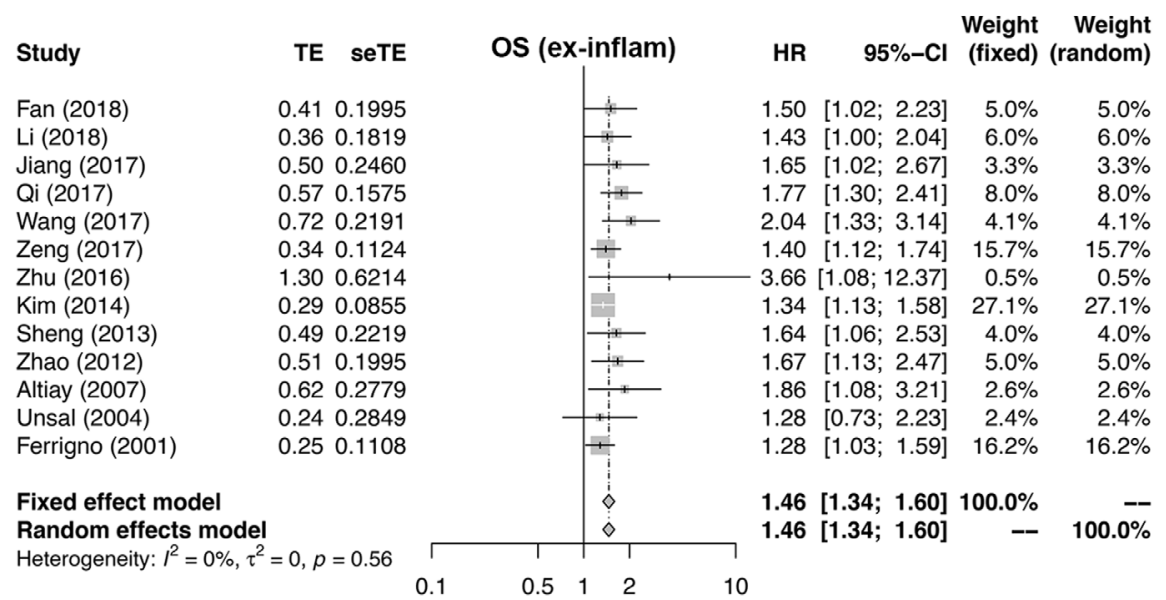

Figure S2 Meta-analysis for assessing the association between plasma fibrinogen and OS in excluded inflammatory disease or coagulation disorders group. OS, overall survival; ex-inflam, analysis in studies excluded inflammatory disease or coagulation disorders. 


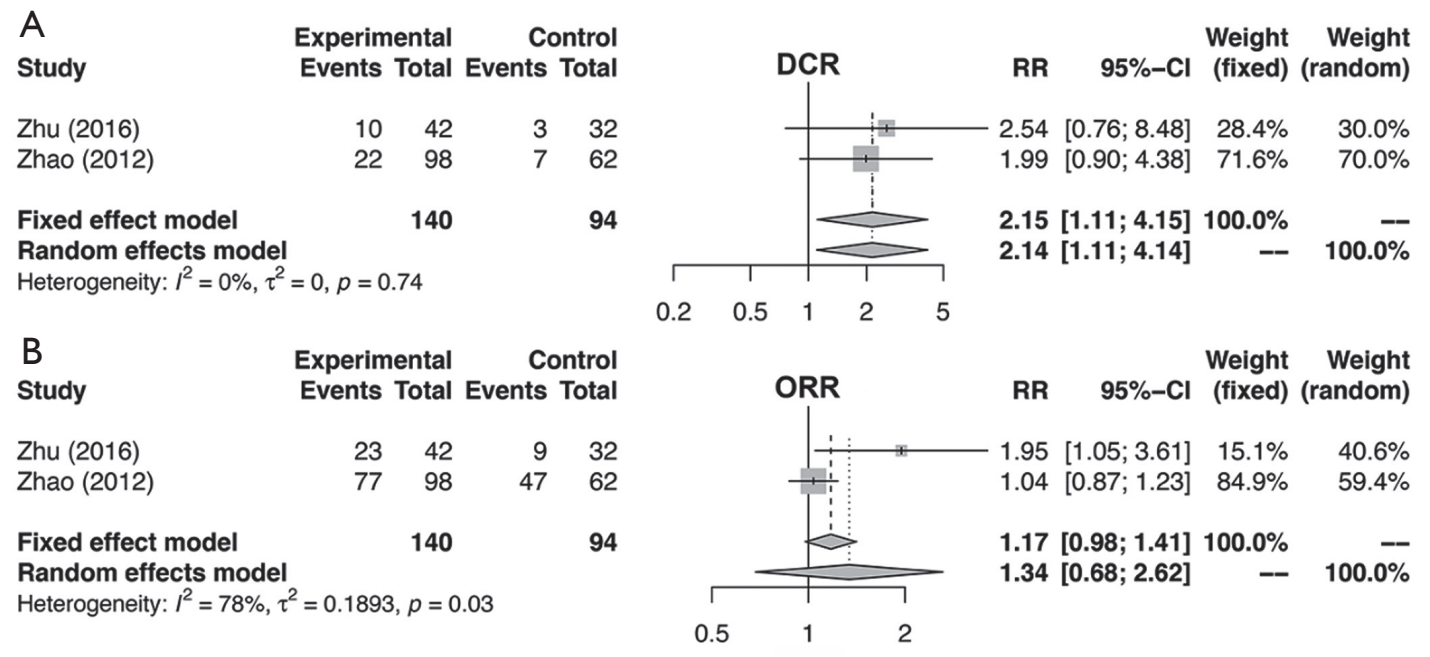

Figure S3 Meta-analysis about DCR (A), ORR (B) estimated by odds ratio. DCR, disease control rate; ORR, objective response rate.

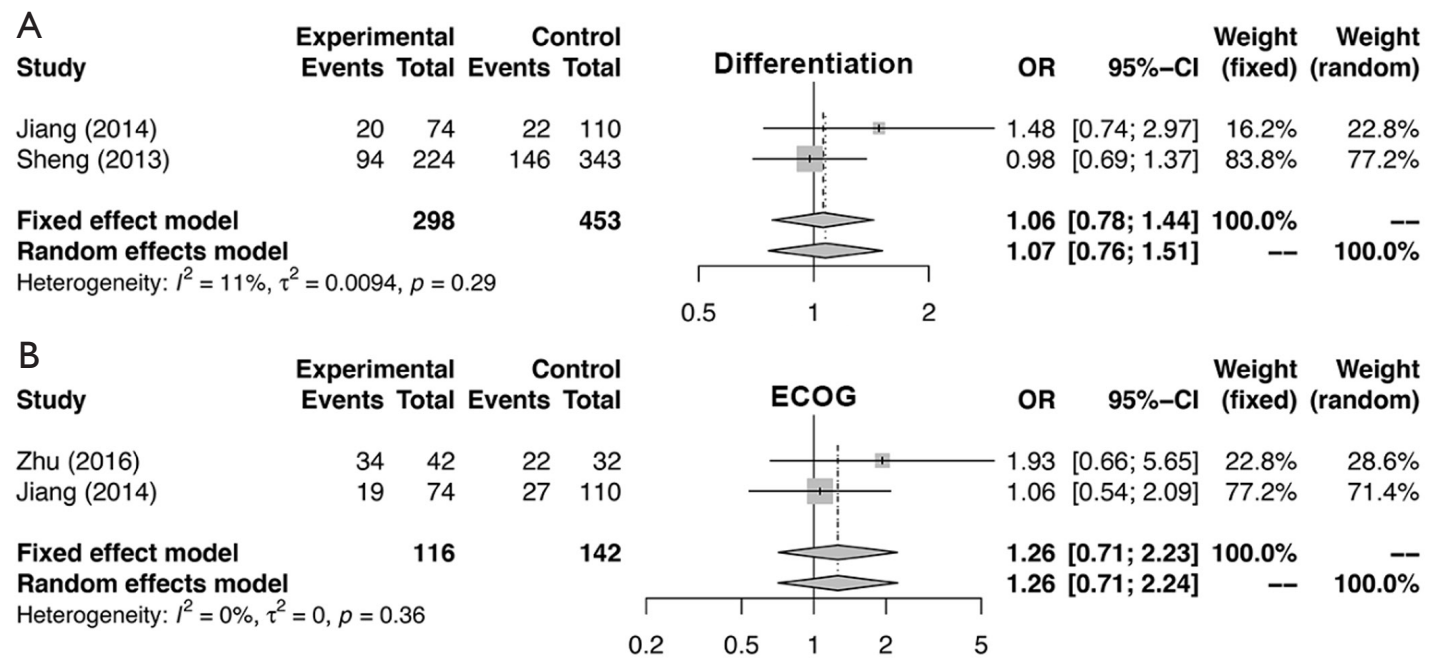

Figure S4 Meta-analysis about differentiation (A) and performance status (B). ECOG, Eastern Cooperative Oncology Group. 


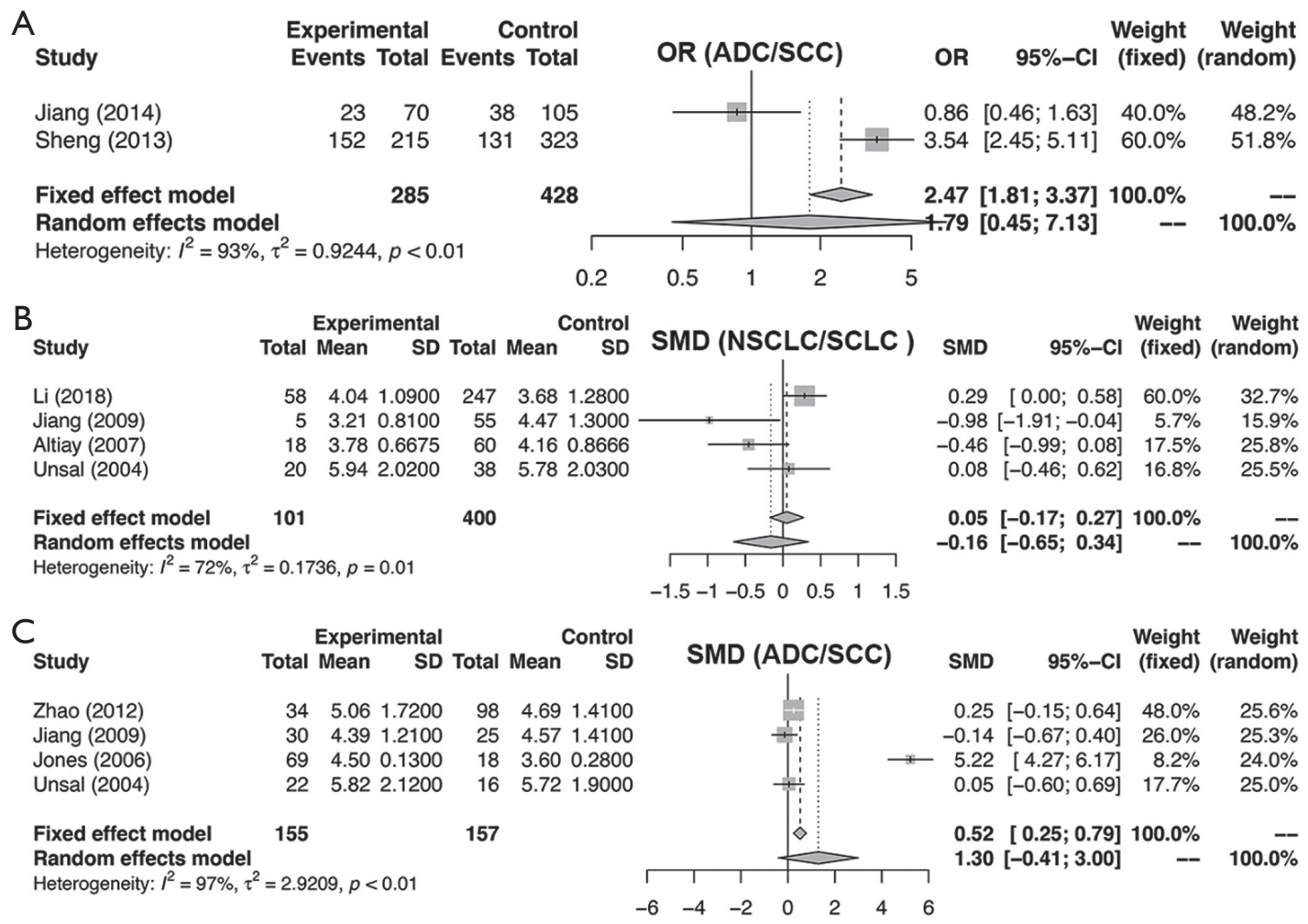

Figure S5 Meta-analysis about histology in ADC vs. SCC (A) estimated by odds ratio, NSCLC vs. SCLC (B), ADC vs. SCC (C) estimated by standardized mean difference. NSCLC, non-small cell lung cancer; SCLC, small cell lung cancer; ADC, adenocarcinoma; SCC, squamous cell carcinoma. 

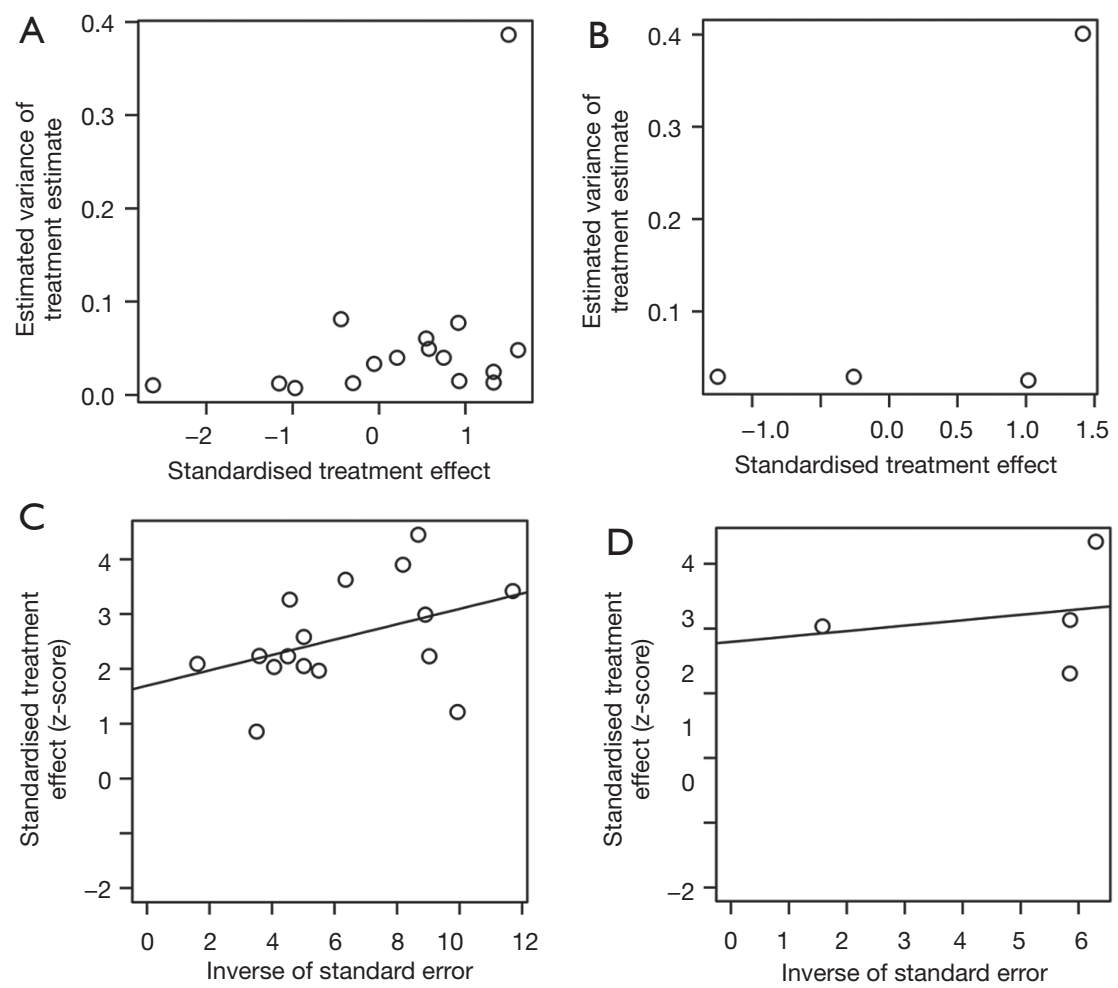

Figure S6 Publication bias of meta-analysis assessing the association between plasma fibrinogen and overall survival and progression-free survival of lung cancer in rank correlation method (A,B) and linear regression method (C,D).
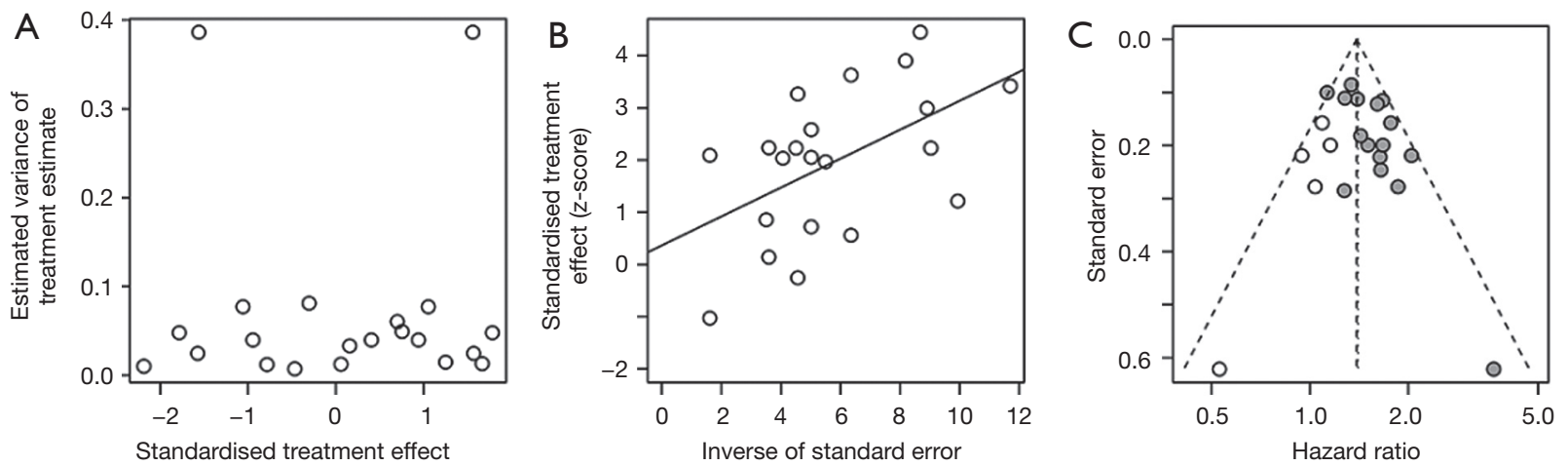

Figure S7 Adjusting the bias using a trim-and-fill method in the association between plasma fibrinogen and overall survival with rank correlation method (A), linear regression method (B) and funnel plot (C). 